\title{
Prominence Disappearance Related to CMEs
}

\author{
E. Hiei \\ Meisei University, 2-1-1, Hodokubo, Hino, Tokyo 191, Japan
}

\begin{abstract}
DB (disparition brusque) events are associated with dynamic phenomena such as a CME, a flare, brightening of a soft X-ray arcade, and soft $\mathrm{X}$-ray dimming, and probably a change of the coronal magnetic field on a large scale. The DB event observed on January 16, 1993 identified with a CME occurred on the solar disk.
\end{abstract}

\section{Introduction}

A DB (disparition brusque) is a signature of a change in the coronal magnetic field surrounding a prominence. A CME (Coronal Mass Ejection), a flare, brightening of soft X-ray arcade, and soft X-ray dimming may all be related to a change of the coronal magnetic field configuration. However, which is a cause of the other event is not known. Several examples of the observations of DB/CME/ flare/soft X-ray brightening/dimming are discussed to find out which is the first event among the other ones.

\section{Observations}

We have studied a relation between the $\mathrm{DB}$ of a polar crown prominence and $\mathrm{CME}$ /soft X-ray brightening/dimming, and found that a CME and a DB occur almost simultaneously, but the brightening of a soft X-ray arcade occurs always after a DB event. It is important to determine which (CME or DB) starts first. However, it is difficult to determine a starting time of the event. A prominence is usually moving up slowly at the beginning and then shows an abrupt change of its upward velocity (Mouradian 1995). The behavior of a CME is also difficult to determine when the CME begins to move up. At the beginning of a CME, it moves up slowly and its intensity is seen to be very weak. The observation of a CME, however, shows that the front of the CME moves faster than that of the lifting prominence (Hundhausen 1998), so it is natural to think that a prominence may not push a CME, but rather a CME may be the cause of a DB.

A good example of a $\mathrm{DB}$, a $\mathrm{CME}$, and brightening of a soft X-ray loop was observed at the southwest limb. A polar crown prominence disappeared on April 30,1993, and a lifting prominence and its related CME were observed with the K-coronameter at Mauna Loa in Hawaii, and soft X-ray brightening was observed with the soft X-ray telescope (SXT) on board the Yohkoh satellite. Figure 1 shows the observed height of the CME (cavity) and the prominence versus time. The velocity of the CME was higher than that of the prominence. A soft X-ray loop brightened up almost 2 hours later than the first appearance of 
the lifting prominence. The SXT loop extended up along the trajectory of the lifting prominence.

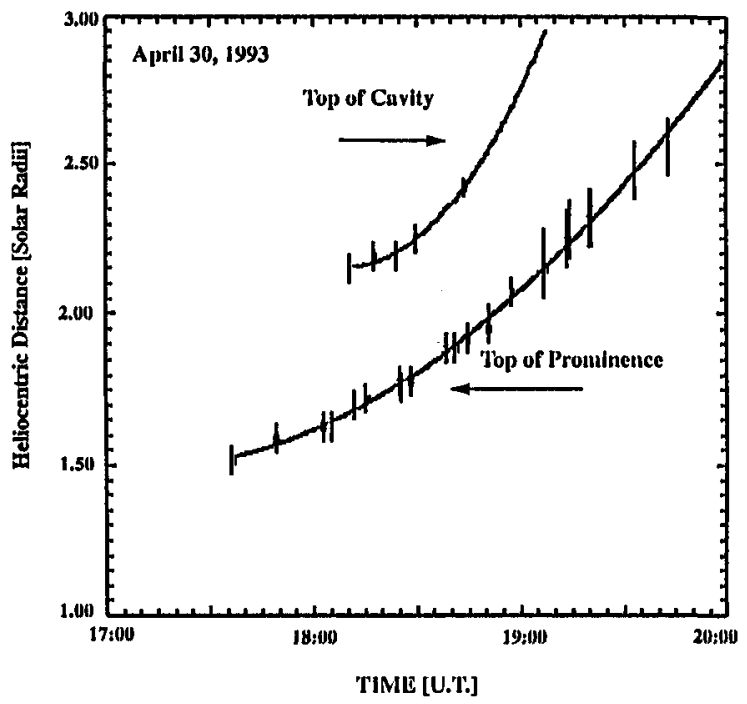

Figure 1. Height change of CME and prominence.

The soft X-ray intensity of the coronal region above the X-ray bright loop showed a dimming, which started at almost the same time as the first appearance of the CME. The X-ray intensity of the dimming region recovered 7 hours later. The dimming seemed to be due to the depletion of coronal matter associated with the CME.

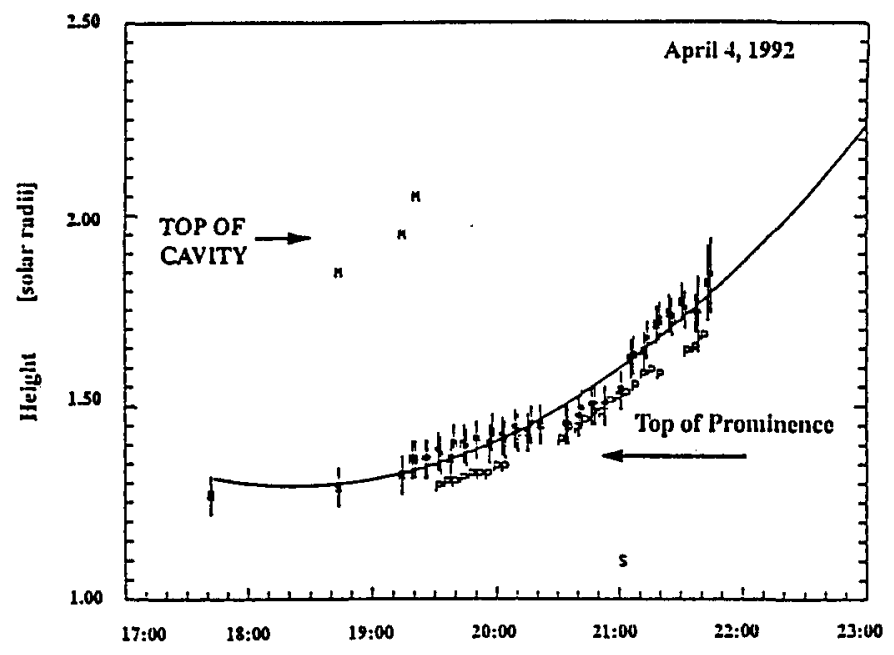

Figure 2. Height change of $\mathrm{CME}$ and prominence. 
A large polar crown prominence erupted on April 4, 1992. The lifting prominence and its related CME were observed at Mauna Loa. Figure 2 shows the height variation of the top of the CME cavity and the lifting prominence versus time. While the $\mathrm{DB}$ and CME were obvious in visible wavelengths, the only significant feature detected in soft $\mathrm{X}$-rays was a faint knot of enhanced emission at approximately 0.11 solar radii above the limb about 3 hours later than the appearance of the prominence. The soft X-ray emission thus occurred below the location of the prominence, and after the acceleration phase of the eruption.

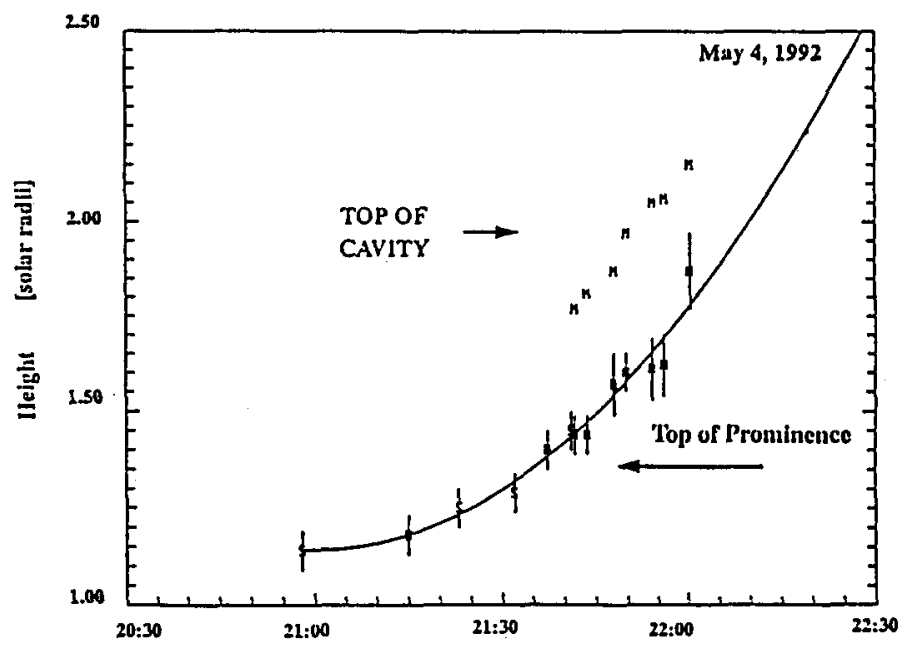

Figure 3. Height change of CME and prominence.

Figure 3 shows the height change of a CME and a prominence versus time on May 4,1992. The outer loop, cavity and central core of the CME were observed with the K-coronameter at Mauna Loa. An outward motion of material was seen in SXT data and might be identified as the front of the CME. Inspection of the $\mathrm{H} \alpha$ data, however, reveals that the mass ejection appeared to arise from active region 7154 near $\mathrm{S} 27 \mathrm{E} 54$, and that the core of the ejected material was associated with a very small filament which erupted from this region. The motion seen in the soft X-ray images, thus, are those of the inner core of the CME and not the outer loop or top of the CME.

A dimming event on January 16,1993 , associated with a DB and soft X-ray brightening, provides good evidence of the CME event observed on the solar disk. The disappearance of the DB was observed between 09:00 UT and 12:30 UT. The dimming started at 12:00 UT and was greatest at 13:30 UT. The duration of the dimming was not determined because the later phase of the dimming was affected by the brightening of soft X-ray loops appearing after the DB.

A particle decrease at the earth, which indicates the presence of an interplanetary CME, was detected at 10:22 UT on January 19, 1993 (Cane 1996, private communication) and, thus, the DB and its associated dimming of January 16 can be identified with a CME observed on the disk. 


\section{References}

Hundhausen, A.J. 1998, Coronal Mass Ejections, in The Many Faces of the Sun, (eds.) K.T. Strong, J.L.R. Saba, B.M. Haisch, and J.T. Schmeltz, Springer-Verlag, New York

Mouradian, Z., Soru-Escaut, I. and Pojoga, S. 1995, Solar Phys., 158, 269 